Doug Geisler, Eva K. Grebel, and Dante Minniti, eds.

\title{
A Space Motion Study of the Globular Cluster M13
}

\author{
L.Chen, J.J.Wang, and J.L.Zhao
}

Shanghai Astronomical Observatory, CAS, Shanghai 200030, P.R.China Chinese National Observatories, CAS, Beijing 100012, P.R.China

\begin{abstract}
Positions and absolute proper motions of 264 stars in the field of about $70^{\prime} \times 70^{\prime}$ around the globular cluster M13 were determined. In the astrometric reduction, three early and three late epoch plates taken with the $40 \mathrm{~cm}$ refractor at Shensan, Shanghai, China, were used, with the time baseline of 73 years. The reduction was done using the central overlapping algorithm, with 5 Hipparcos stars and 38 Tycho-2 stars used as reference stars. Based on the reduced proper motion data, the membership probabilities of the sample stars were determined. An absolute proper motion of the cluster of $1.49 \pm 0.36$ mas $\mathrm{yr}^{-1}$ in right ascension and $3.06 \pm 0.35$ mas $\mathrm{yr}^{-1}$ in declination was obtained. From this proper motion, together with distance and radial velocity data, we derived the space motion of M13 and via numerical integration also the orbit of the cluster in the Galaxy.
\end{abstract}

\section{Observations and the proper motion reduction}

The six plates of M13 used in this work were taken with the $40 \mathrm{~cm}$ refractor $(\mathrm{f}=6895 \mathrm{~mm})$ without any filter and roughly in the B-passband. From PDS measurements 292 stars were extracted in these plates with their rectangular coordinates $\mathrm{x}$ and $\mathrm{y}$ determined. Among these stars, 43 were Tycho- 2 stars including 5 Hipparcos stars. For these 5 Hipparcos stars, their astrometric data in the HIPPARCOS Catalogue were used in the astrometric reduction. The sample stars covered a field of about $70^{\prime} \times 70^{\prime}$ around the center of the globular cluster. The astrometric reduction was performed by a central overlapping method. Finally, accurate positions and proper motions of 264 stars around M13 were obtained. Figure 1, with different symbols representing different membership probabilities $p$, gives the distribution of positions of these 264 stars and also a vector point diagram of the proper motions in the field of M13, which indicates a clear separation of cluster and field stars. To estimate the cluster membership probabilities of the 264 stars by means of the maximum likelihood method we used the formalism similar to that of Shu, Fu \& Zhao (1995), but in our model we adopted a Gaussian distribution instead of an exponential for the position distribution of the cluster stars.

From the above reductions and some detailed analysis of the magnitudeand color-dependent trends in the proper motions of cluster stars we finally

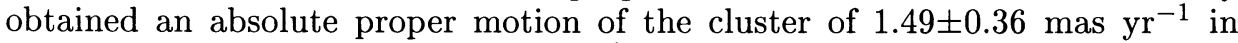

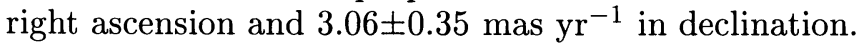



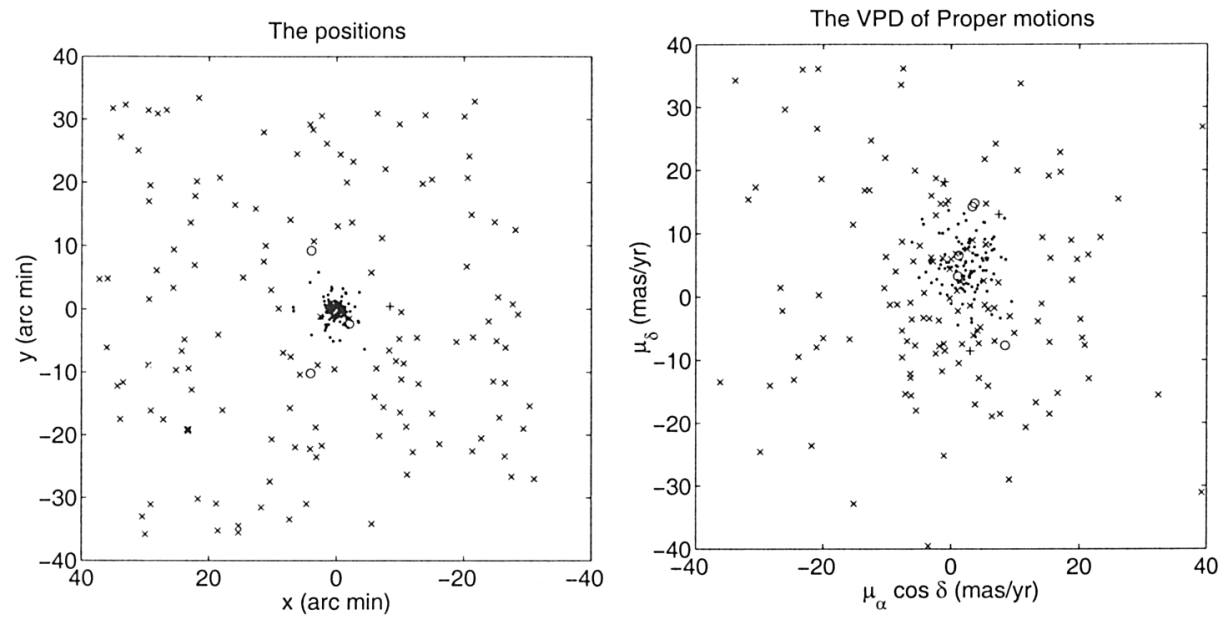

Figure 1. Left: Distribution of positions of 264 stars around M13, Right: Vector point diagram of the proper motions $\mathrm{x}: p<0.1,+$ : $0.1 \leq p<0.5$, o: $0.5 \leq p<0.9, \bullet: 0.9 \leq p<1.0$ , where $p$ is the proper motion.

\section{The space motion of M13 and Discussions}

From the adopted mean absolute proper motion of M13, together with its distance and radial velocity we calculated the present space velocity of this cluster in respect to the galactic standard of rest. From its present position and velocity, we obtained the orbit of M13 integrated over 10 Gyr backwards based on the Galactic potential of Allen \& Santillan (1991). Table 1 gives some orbital parameters of M13, where $\mathrm{R}$ is the Galactic distance, $\mathrm{z}$ the distance from the Galactic plane, $\mathrm{v}$ the space velocity $(\mathrm{t}=0$ means at the present time), e the eccentricity, i the inclination angle with respect to the Galactic plane, $T$ is the period, and value $N_{r e v}$ the number of complete revolutions. The angular momentum of the cluster $J_{z}=41 \mathrm{kpc} \mathrm{km} \mathrm{s}{ }^{-1}$, and the orbital energy per unit mass $E / m=-108104 \mathrm{~km}^{2} \mathrm{~s}^{-2}$. Figure 2 gives the projected orbit of M13 on the meridian plane and on the Galactic plane as well.

Table 1. Orbital parameters of the globular cluster M13

\begin{tabular}{cccccc}
\hline \multicolumn{2}{c}{ Geometry } & \multicolumn{2}{c}{ Velocity } & \multicolumn{2}{c}{$\mathrm{J}$ and incl. } \\
\hline$R_{t=0}$ & $8.7 \mathrm{kpc}$ & $v_{t=0}$ & $297 \mathrm{~km} \mathrm{~s}^{-1}$ & $J_{\text {mean }}$ & $2395 \mathrm{kpc} \mathrm{km} \mathrm{s}^{-1}$ \\
$R_{\text {max }}$ & $21.8 \mathrm{kpc}$ & $v_{\text {max }}$ & $342 \mathrm{~km} \mathrm{~s}^{-1}$ & $J_{\text {max }}$ & $2799 \mathrm{kpc} \mathrm{km} \mathrm{s}$ \\
$R_{\text {min }}$ & $7.3 \mathrm{kpc}$ & $v_{\text {min }}$ & $101 \mathrm{~km} \mathrm{~s}^{-1}$ & $J_{\text {min }}$ & $2198 \mathrm{kpc} \mathrm{km} \mathrm{s}^{-1}$ \\
$e$ & 0.50 & $v_{\text {mean }}$ & $234 \mathrm{~km} \mathrm{~s}^{-1}$ & $i_{\text {mean }}$ & $89^{\circ} .0$ \\
$z_{\text {max }}$ & $21.8 \mathrm{kpc}$ & $T_{\text {min }}$ & $0.47 \mathrm{Gyr}^{\circ}$ & $i_{\text {max }}$ & $88^{\circ} .9$ \\
$z_{\text {min }}$ & $-21.8 \mathrm{kpc}$ & $N_{\text {rev }}$ & 21.5 & $i_{\text {min }}$ & $89^{\circ} .2$ \\
\hline
\end{tabular}



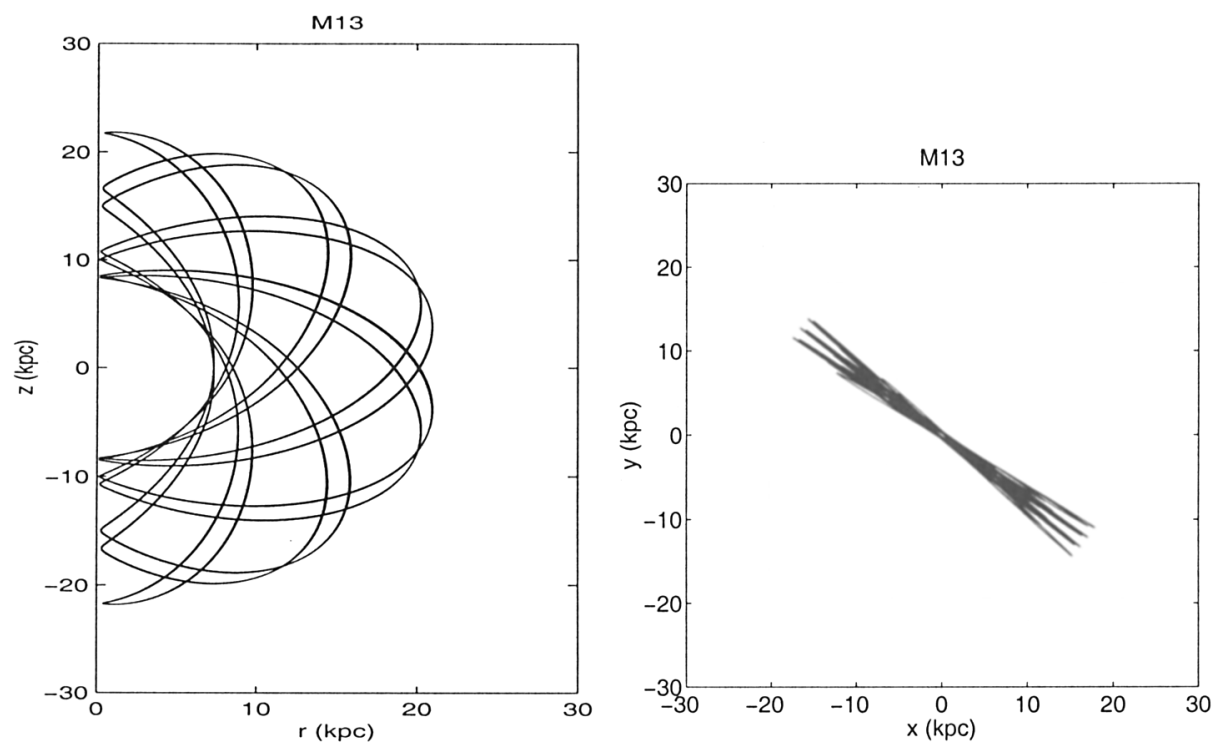

Figure 2. Orbit of M13 projected onto the meridianal plane (left) and the Galactic plane (right), respectively.

Our orbit of globular cluster M13 differs significantly from those of Dauphole et al. (1996) and Dinescu, Girard \& van Altena (1999) based on the absolute proper motion study by Cudworth \& Hanson (1993). The discrepancy is mainly due to the difference of proper motion results. Our maximum plate epoch difference is nearly the same as that of Cudworth \& Monet (1979), and our reduction was based on Hipparcos and the Tycho- 2 catalogues which brought forth in absolute proper motion determination the minimum systematic errors from the reference frame. The orbital parameters derived from this work should be more reliable. From our result, M13 is characterized as a typical outer-halo globular cluster, with its apogalactic distance of $22 \mathrm{kpc}$, its orbit nearly vertical to the galactic plane, with the angular momentum $L_{z}$ very small. And according to its $\Theta$ velocity component in the galactic cylindrical coordinate system this cluster has a small retrograde galactic orbit.

Acknowledgments. This research was partially supported by NKBRSF19990754 and National Natural Sciences Foundation under grant 19833010.

\section{References}

Allen, C., \& Santillan, A. 1991, Rev. Mexicana Astron. Astrof., 22, 255

Dauphole, B., Geffert, M., \& Colin, J. 1996, A\&A, 313, 119

Dinescu, D.I., Girard, T.M., \& van Altena, W.F. 1999, AJ, 117, 1792

Cudworth, K.M., \& Hanson, R.B. 1993, AJ, 105, 168

Cudworth, K.M., \& Monet, D.G. 1979, AJ, 84, 774

Shu, C.C., Fu, C.Q., \& Zhao, J. L. 1995, Acta Astrophysica Sinica, 15, 205 